\title{
MICROLENSING EVENTS BY PROXIMA CENTAURI IN 2014 AND 2016: OPPORTUNITIES FOR MASS DETERMINATION AND POSSIBLE PLANET DETECTION*
}

\author{
Kailash C. Sahu ${ }^{1}$, Howard E. Bond ${ }^{1,2}$, Jay Anderson ${ }^{1}$, And Martin Dominik ${ }^{3,4}$ \\ ${ }^{1}$ Space Telescope Science Institute, 3700 San Martin Drive, Baltimore, MD 21218, USA; ksahu@ stsci.edu, jayander@ stsci.edu \\ ${ }^{2}$ Department of Astronomy and Astrophysics, Pennsylvania State University, University Park, PA 16802, USA; heb11@ psu.edu \\ ${ }^{3}$ SUPA, School of Physics and Astronomy, University of St. Andrews, North Haugh, St. Andrews KY16 9SS, UK; md35@ @st-andrews.ac.uk \\ Received 2013 January 24; accepted 2013 December 31; published 2014 January 31
}

\begin{abstract}
We have found that Proxima Centauri, the star closest to our Sun, will pass close to a pair of faint background stars in the next few years. Using Hubble Space Telescope (HST) images obtained in 2012 October, we determine that the passage close to a mag 20 star will occur in 2014 October (impact parameter 1".6), and to a mag 19.5 star in 2016 February (impact parameter 0.'5). As Proxima passes in front of these stars, the relativistic deflection of light will cause shifts in the positions of the background stars of $\sim 0.5$ and 1.5 mas, respectively, readily detectable by HST imaging, and possibly by Gaia and ground-based facilities such as the Very Large Telescope. Measurement of these astrometric shifts offers a unique and direct method to measure the mass of Proxima. Moreover, if Proxima has a planetary system, the planets may be detectable through their additional microlensing signals, although the probability of such detections is small. With astrometric accuracies of 0.03 mas (achievable with HST spatial scanning), centroid shifts caused by Jovian planets are detectable at separations of up to 2".0 (corresponding to 2.6 AU at the distance of Proxima), and centroid shifts by Earth-mass planets are detectable within a small band of 8 mas (corresponding to $0.01 \mathrm{AU}$ ) around the source trajectories. Jovian planets within a band of about 28 mas (corresponding to $0.036 \mathrm{AU}$ ) around the source trajectories would produce a brightening of the source by $>0.01 \mathrm{mag}$ and could hence be detectable. Estimated timescales of the astrometric and photometric microlensing events due to a planet range from a few hours to a few days, and both methods would provide direct measurements of the planetary mass.
\end{abstract}

Key words: astrometry - gravitational lensing: micro - planetary systems - stars: individual (Proxima Centauri)

\section{PROXIMA CENTAURI: OUR NEAREST NEIGHBOR}

Proxima Centauri ( $\alpha$ Centauri C, V645 Cen, GJ 551; Hipparcos parallax $771.64 \pm 2.60$ mas) is the nearest known star, lying $1.30 \mathrm{pc}$ from the Sun. It is located at a projected angular separation of 2.2 and a linear distance of $0.07 \mathrm{pc}(14,500 \mathrm{AU})$ from the bright and slightly more distant binary $\alpha$ Cen A+B. The distance, proper motion, and radial velocity (RV) of Proxima are closely similar to those of $\alpha$ Cen $\mathrm{A}+\mathrm{B}$, showing that the system forms a wide triple (Wertheimer \& Laughlin 2006); however, Proxima's large separation from $\alpha$ Cen A+B means that it has evolved effectively as a single star. Proxima is a low-mass, M5 Ve dwarf and flare star, with an apparent magnitude of $V=11.05$, a RV of $-22.4 \mathrm{~km} \mathrm{~s}^{-1}$ (Torres et al. 2006), and a proper motion of $33^{\prime \prime} 8526 \pm 0.0026 \mathrm{yr}^{-1}$ in position angle (P.A.) $281: 461 \pm 0.036$, as measured by Hipparcos (van Leeuwen 2007; also see Benedict et al. 19995).

\subsection{Mass of Proxima Centauri}

Proxima, as an M dwarf, represents the most common type of star. Proxima's radius is known from Very Large Telescope Interferometry observations to be $0.145 R_{\odot} \pm 0.011 R_{\odot}$ (Ségransan et al. 2003). But, as an effectively isolated star, Proxima's mass can only be estimated from mass-luminosity $(M-L)$ relations as $\sim 0.12 M_{\odot}$.

\footnotetext{
* Based in part on observations made with the NASA/ESA Hubble Space Telescope, obtained at the Space Telescope Science Institute, which is operated by the Association of Universities for Research in Astronomy, Inc., under NASA contract NAS 5-26555.

4 Royal Society University Research Fellow.

5 Benedict et al. (1999) give a proper-motion P.A. of 78.46, evidently measuring it from north through west instead of the conventional direction.
}

A star's mass is its most important physical parameter, being the primary determinant of its temperature, radius, luminosity, lifetime, and ultimate fate. The only directly measured stellar masses come from double-lined eclipsing systems and visual binaries. For dM stars like Proxima, the observational situation is particularly poor, with less than a dozen double-lined eclipsing systems known that have primary masses of $<0.6 M_{\odot}$ (e.g., Kraus et al. 2011). Moreover, the eclipsing systems are not representative of field $\mathrm{dM}$ stars, because synchronous rotation causes stellar activity, starspots, and inflated radii. The masses of single low-mass stars have to be inferred largely from theoretical $M-L$ relations, which suffer from poorly understood opacities and interior structure (e.g., Chabrier \& Baraffe 1997, 2000).

\subsection{Planets Around Proxima Centauri}

Ground-based RV measurements have recently led to discovery of a $m \sin i=1.1 M_{\text {Earth }}$ planet orbiting $\alpha$ Cen B at a distance of $0.04 \mathrm{AU}$ (Dumusque et al. 2012). However, all attempts to detect planets around Proxima Centauri have so far been unsuccessful. M dwarfs are generally not optimal for searching for planets through RV techniques, since a large fraction of them have surface activity, which increases RV jitter (e.g., Campbell et al. 1991; Wright 2005). However, Proxima itself has a relatively small RV scatter of $3.1 \mathrm{~m} \mathrm{~s}^{-1}$, and RV techniques have been used extensively to search for planets around it. These data exclude the presence of any planet in a circular orbit with $m \sin i>1 M_{\text {Neptune }}$ at orbital separations of $a<1$ AU (Endl \& Kürster 2008). However, this does not rule out the presence of larger planets whose orbital plane may be close to face-on. No planetary transits have been reported for Proxima. Astrometric techniques have also been unsuccessful in detecting any planets 
around Proxima, which excludes the presence of any planet with mass $>0.8 M_{\text {Jup }}$ in the period range 1 day $<P<1000$ days, corresponding to orbital separations of 0.01-1 AU (Benedict et al. 1999).

\section{PREDICTING ASTROMETRIC MICROLENSING EVENTS DUE TO NEARBY STARS}

In this paper we discuss an alternative method for measuring masses of nearby single stars and for searching for planetary companions, and an upcoming opportunity to apply this method to Proxima Centauri. The special importance of microlensing events produced by very nearby stars was emphasized by Paczyński (1996). For such events the term "mesolensing" has been suggested (Di Stefano 2008), because the large angular Einstein radii of nearby stars, coupled with their typically large proper motions, yield relatively large probabilities of such events occurring. Searches for upcoming close stellar passages of high-proper-motion stars near background sources have been carried out by Salim \& Gould (2000) and Proft et al. (2011), who have published lists of candidate events (see also Lépine \& Di Stefano 2012, who discuss a specific event).

In the case of microlensing events due to nearby stars, the foreground star will almost always be much brighter than the background source, making it difficult to measure its astrometric shift of the fainter background star. The contamination from the foreground star reduces when the lens-source separation is larger, but then the astrometric signal also reduces, making the measurement again difficult. Measuring the astrometric signal will be easiest if we can find a situation where the centroid shift is large even when the lens-source angular separation is large. Such a situation arises for the very nearest stars, for which the angular Einstein radii are the largest.

To search for such very favorable events, we started with the Luyten Half Second (LHS) catalog (Luyten 1979), a compilation of all known stars with proper motions greater than $0^{\prime} .5 \mathrm{yr}^{-1}$. The coordinates in the LHS catalog are only approximate, so we used improved positions and proper motions determined by Lépine \& Shara (2005; mostly for northern-hemisphere stars), and the revised positions and proper motions published by Bakos et al. (2002) for the remainder. Unlike the previous studies, we took the parallaxes of the LHS stars into account, if they are available in the SIMBAD database, since parallax significantly alters the circumstances of the events for very nearby stars. We then projected the positions of all $\sim 5000$ LHS stars forward over the next $40 \mathrm{yr}$ and searched for close passages (impact parameter $<2^{\prime \prime}$ ) near background stars contained in the GSC 2.3 catalog 6 (Lasker et al. 2008).

\section{THE PROXIMA CENTAURI EVENTS OF 2014 AND 2016}

Of the events that we have found will occur in the next few years, one of the most interesting is the close passage of Proxima Centauri in front of a pair of background source stars of $V$ magnitudes 20 and 19.5, separated by 4 ".3. Closest approaches will occur in 2014 and 2016, respectively. Figure 1 shows images

\footnotetext{
6 http://gsss.stsci.edu/Catalogs/GSC/GSC2/gsc23/gsc23_release_notes.htm. The Guide Star Catalogue-II is a joint project of the Space Telescope Science Institute and the Osservatorio Astronomico di Torino. Participation of the Osservatorio Astronomico di Torino is supported by the Italian Council for Research in Astronomy. Additional support is provided by European Southern Observatory, Space Telescope European Coordinating Facility, the

International Gemini project, and the European Space Agency Astrophysics Division.
}
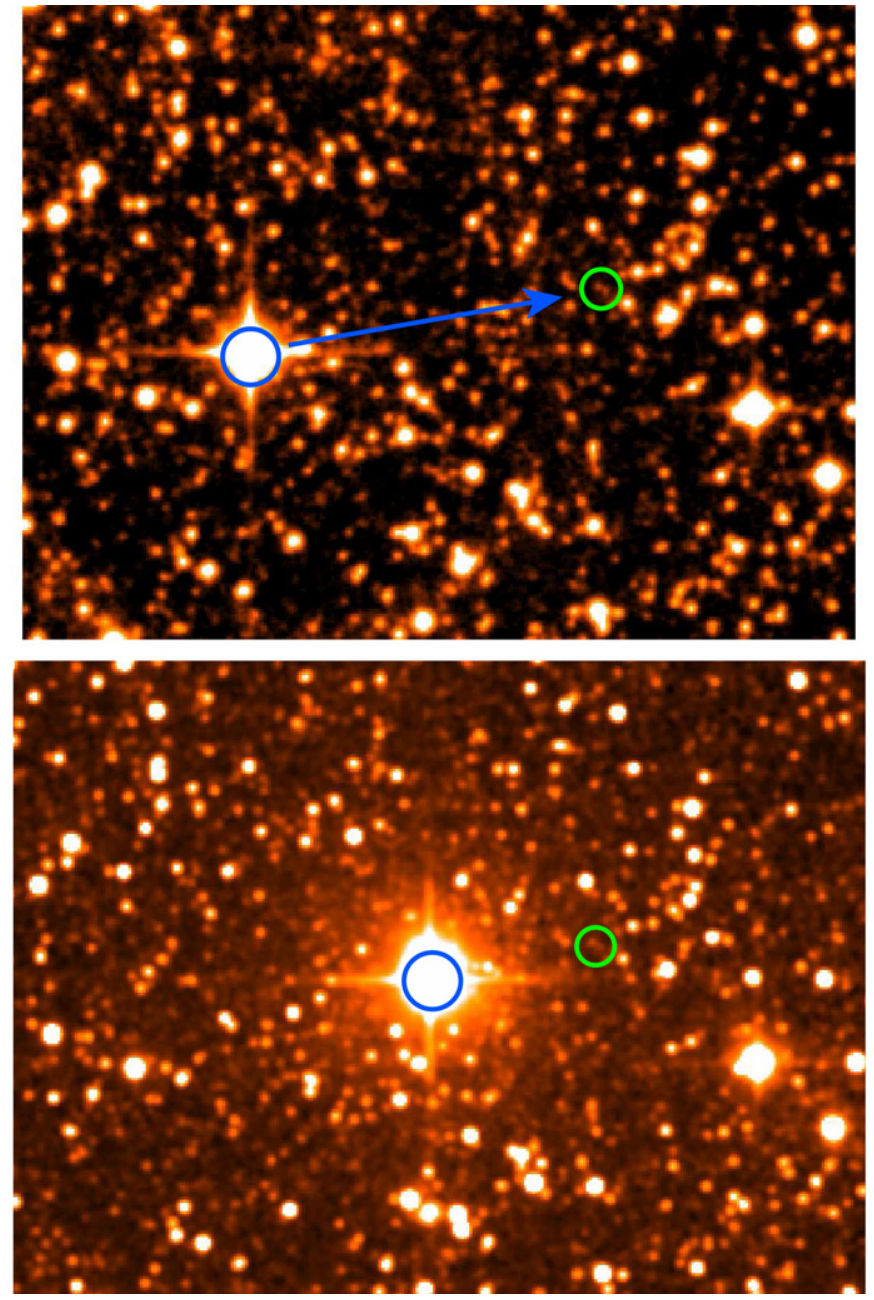

Figure 1. Top: Proxima Centauri field in 1997, taken from the near-IR Digitized Sky Survey. The image size is $300^{\prime \prime} \times 220^{\prime \prime}$ with north at the top and east on the left. Proxima is encircled in blue, and is moving to the west-northwest. Bottom: Proxima field in 2000, taken in the 2MASS survey. In both images the pair of faint background stars that will be microlensed in 2014 and 2016 is within the green circles on the right.

of the Proxima field from the Digitized Sky Survey ${ }^{7}$ infrared (IR) and Two Micron All Sky Survey (2MASS; Cutri et al. 2003) surveys, taken in 1997 and 2000, respectively. Proxima is the bright star to the left, marked with a blue circle, and moving rapidly to the right (west-northwest). A second green circle marks the pair of background stars.

Although it might appear from a cursory inspection of the ground-based images in Figure 1 that close passages must occur frequently, passages as close as $\sim 1^{\prime \prime} .5$ are actually rare. Moreover, Proxima is exceptional in having an Einstein ring radius as large as 28 mas, because it is so close to the Earth. For more typical proper-motion stars at considerably larger distances than Proxima, only extremely close passages $\left(<0^{\prime} .3\right)$ are of interest from a microlensing standpoint-and they would generally suffer considerably from large differences in brightnesses.

To refine the impact parameters and times of closest approach, we obtained Hubble Space Telescope (HST) images of the field on 2012 October 1. Observations were taken with the UVIS channel of the Wide Field Camera 3 (WFC3) in the F475W,

\footnotetext{
The Digitized Sky Survey was produced at the Space Telescope Science Institute under U.S. Government grant NAG W-2166.
} 
Table 1

Details of the Source Stars

\begin{tabular}{|c|c|c|c|}
\hline Parameter & Source 1 & Source 2 & Proxima $^{a}$ \\
\hline R.A. (J2000) & $14: 29: 34.693$ & $14: 29: 34.268$ & \\
\hline Decl. (J2000) & $-62: 40: 33.46$ & $-62: 40: 34.91$ & \\
\hline $\mathrm{F} 475 \mathrm{~W}$ (“ $B$ ") & 21.26 & 20.55 & 12.03 \\
\hline F555W (" $V ")$ & 20.36 & 19.89 & 11.33 \\
\hline F606W ("wide-V") & 19.61 & 19.29 & $\left(10.44^{\mathrm{a}}\right)$ \\
\hline F814W (“' $I ”)$ & 17.78 & 17.93 & $\left(7.25^{\mathrm{a}}\right)$ \\
\hline \multirow[t]{2}{*}{ Date of closest approach } & $2014.80 \pm 0.03$ & $2016.16 \pm 0.03$ & \\
\hline & (2014 Oct $20 \pm 10$ days) & (2016 Feb $26 \pm 10$ days) & \\
\hline Impact parameter & $1^{\prime \prime} 6 \pm 0^{\prime \prime} 1$ & $0^{\prime \prime} 5 \pm 0^{\prime \prime} 1$ & \\
\hline
\end{tabular}

Note. ${ }^{\text {a }}$ The magnitudes of Proxima in the F606W and F814W filters are determined from saturated images which are uncertain by $\pm 0.5 \mathrm{mag}$. All other magnitudes have uncertainty of $\pm 0.05 \mathrm{mag}$.

F555W, F606W, and F814W filters. Since the background stars are $>8$ mag fainter than Proxima, we obtained two sets of observations, a short one with an exposure time of $0.5 \mathrm{~s}$ (the minimum allowed exposure time with WFC3) in each filter and a long one with an exposure time of 100 to 200 s. An extra set of short and long exposures were obtained in the F555W filter, which were dithered by about 4 " with respect to the first set. The two long exposures obtained in the F555W filter were used to produce a cosmic-ray cleaned image of the field. Since Proxima is heavily saturated in the longer exposures, care was taken to choose a telescope orientation such that neither the diffraction spikes nor the charge bleeding from Proxima would affect the background sources. Proxima was not saturated in the short exposures in the F475W and F555W filters, but was slightly $(\lesssim 30 \%)$ saturated in the F606W and F814W filters. The short exposures were used for photometry of Proxima itself, and the long exposures were used for photometry of the background stars. Even though we have used a large aperture to take the charge bleeding into account in our photometry in the saturated images (Gilliland 1994) in the F814W and F606W filters, the photometric uncertainties in these filters are expected to be higher. The photometric magnitudes for Proxima (on the Vega-mag system) and the source stars along with the estimated errors are listed in Table 1 . We also examined the 2MASS source catalog for the IR magnitudes of the sources. The 2MASS catalog shows a single source at this position, with magnitudes of $J=16.089, H=15.436$, and $K_{s}=15.764$, and uncertainties of about $\pm 0.07 \mathrm{mag}$. These magnitudes most likely represent the combined light of both background sources.

Figure 2 shows our long-exposure HST image taken in the F555W $(V)$ filter. Also shown is the future path of Proxima (green line), calculated using the Hipparcos proper motion and parallax (ESA 1997), and taking into account the stellar positions as observed by HST on 2012 October 1 . The uncertainty in Proxima's path is less than the width of the line during the time interval shown here. As the figure shows, Proxima will actually pass between the two background stars labeled as "Source 1 " and "Source 2," affording two independent opportunities to measure the relativistic light deflection and search for effects of planetary companions. The closest passages will occur in 2014 October, with an impact parameter of $1^{\prime \prime} .6 \pm 0$ "' 1 , and in 2016 February, with an impact parameter of $0{ }^{\prime} .5 \pm 0$.' 1 . This prediction is confirmed through a second set of HST observations that we obtained in 2013 March. Details of the predicted close encounters are given in Table 1. (We have an approved program to obtain further $H S T$ observations at 10 different epochs during 2014-2017. The predictions given in Table 1

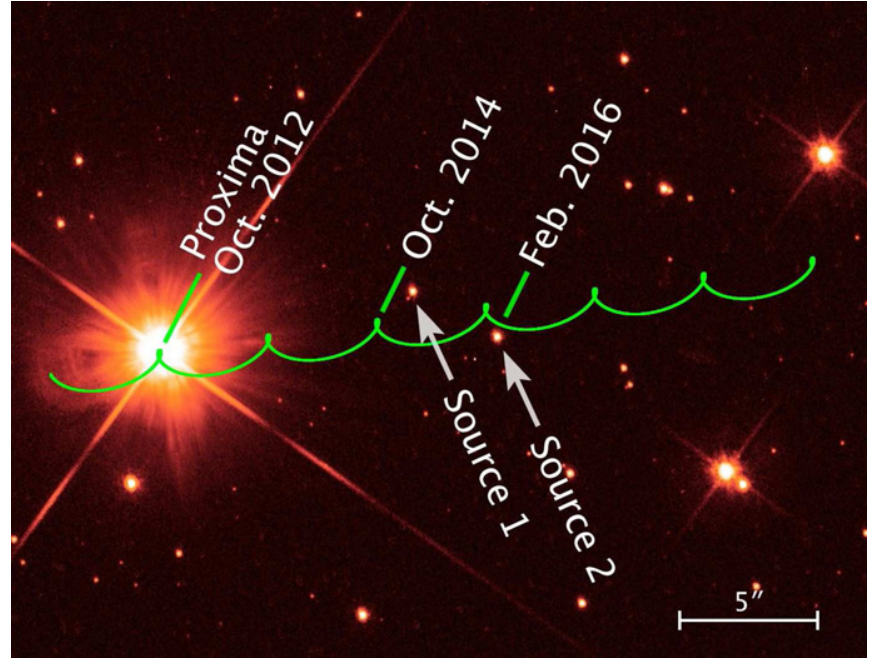

Figure 2. Proxima Centauri field as observed with HST/WFC3 on 2012 October 1 in the F555W ( $V$ ) filter, with north up and east to the left. The two faint background sources are labeled "Source 1" and "Source 2." The future path of Proxima is shown in green, taking into account proper motion and parallax. Considering the facts that (1) the proper motion of Proxima has an accuracy of

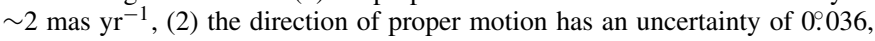
and (3) Proxima was observed by HST in 2012 October, the uncertainty in the proper motion is less than the width of the trajectory during the time interval shown in the figure. The locations and dates of closest approach of Proxima to the two background sources are indicated. The closest approaches to the two background stars will occur in 2014 October and 2016 February, at separations of $1^{\prime \prime} .6 \pm 0$ ". 1 and $0 . .5 \pm 0^{\prime \prime} .1$, respectively (see Table 1 ). Note that there is an additional faint source about 0.3 southeast of Source 1, which is about 2.7 mag fainter than Source 1 in the F555W filter.

will improve as we obtain further $H S T$ observations, and the improved predictions will be made available at this Web site: http://www.stsci.edu/ ksahu. The exact time of the future $H S T$ observations will be adjusted based on results obtained from previous observations, in order to maximize the scientific return.)

We note from Table 1 that the colors of both sources are similar to or bluer than Proxima, but they are at least 8.5 mag fainter than Proxima. That would imply that the distances to these source stars are $>60 \mathrm{pc}$, much larger than the distance to Proxima. In our current analysis of predicting the closest approaches, we have ignored the parallax and proper motion of the source stars, which are expected to be small. This can be rectified if our future $H S T$ observations show any parallax or proper motion. 


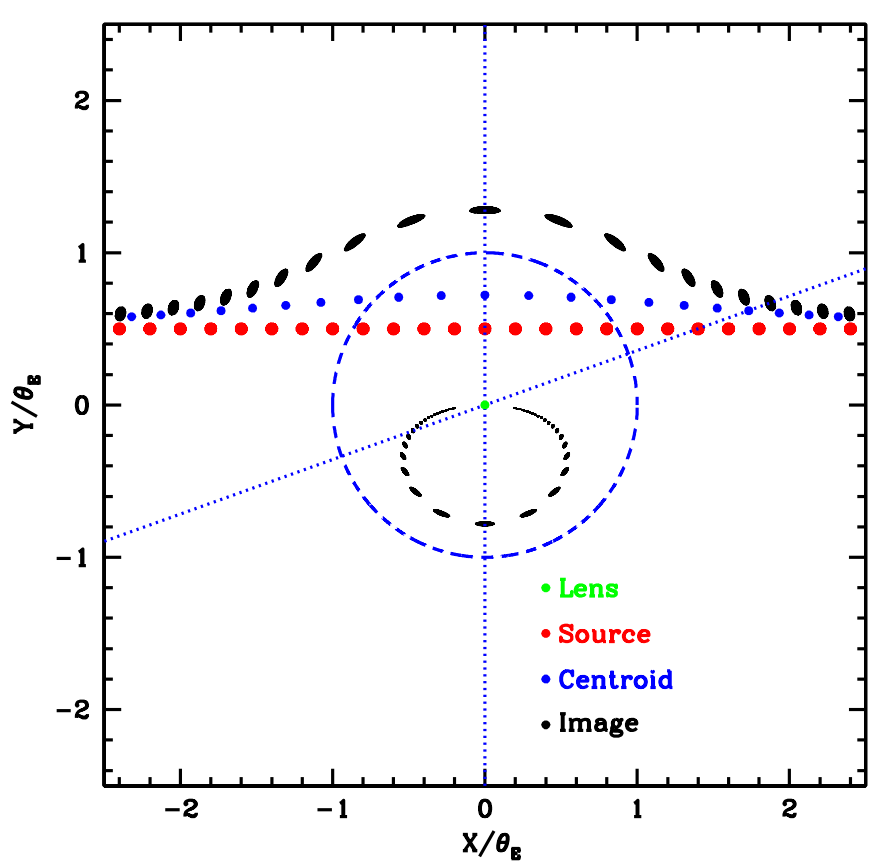

Figure 3. This figure shows how the apparent positions and the sizes of the images change at various stages of a microlensing event. In this geometry the position of the lens, indicated by a green dot, is fixed, and the red dots show the actual positions of the source. The black ellipsoidal points show the images of the source as it passes close to the lens in the plane of the sky. The dashed circle is the Einstein ring of the lens. At any instant, the source, the lens and the two images lie on a straight line as shown by the dotted blue lines. The centroid of the images are indicated by the blue dots, which are clearly shifted with respect to the source positions due to microlensing. The maximum centroid shift occurs when the lens-source separation is $\sqrt{2} \theta_{E}$; one such case is shown by a blue dotted line (cf. Paczyński 1996).

\section{MEASURING THE MASS OF PROXIMA CENTAURI THROUGH ASTROMETRIC MICROLENSING}

When a background star (the source) passes within or close to the Einstein ring of a foreground object (the lens), it splits into two images and the combined brightness exceeds that of the source star as depicted in Figure 3. As explained in more detail later, the sources will pass several Einstein ring radii from Proxima itself, but could pass very close to or within the Einstein ring of a planet. We provide a general formalism here, which is valid for small as well as large impact parameters. (For more details, see Schneider et al. 1992; Paczyński 1996; Dominik \& Sahu 2000.)

The angular radius of the Einstein ring of a point lens can be written as

$$
\theta_{\mathrm{E}}=\left(\frac{4 G M}{c^{2} d_{\pi}}\right)^{1 / 2},
$$

where $M$ is the lens mass and $d_{\pi}$ is the parallax distance of the lens as measured with respect to the more distant source, given by

$$
\frac{1}{d_{\pi}}=\frac{1}{D_{L}}-\frac{1}{D_{S}},
$$

$D_{L}$ and $D_{S}$ being the distances from the Earth to the lens and the source, respectively.

If the angular separation between the lens and the (undeflected) source is $\Delta \theta$, then the two images of the source will be located at separations of

$$
\theta_{ \pm}=0.5\left[u \pm \sqrt{u^{2}+4}\right] \theta_{\mathrm{E}}
$$

relative to the position of the lens, and

$$
u \equiv \Delta \theta / \theta_{\mathrm{E}}
$$

Note that the source, the lens, and the two images of the source all lie on a straight line; the positive sign implies the same side as the source from the lens, and the negative sign implies the opposite side. The angular distances of the two images from the original position of the source are given by $\left(\theta_{ \pm}-\Delta \theta\right)$.

Figure 3 schematically shows images as the source (shown by the red dots) passes though the Einstein ring (blue circle) of the lens. The two images are shown in black, one outside the Einstein ring (major image), and the other inside (minor image).

The amplifications of the two images of the source are given by

$$
A_{ \pm}=0.5\left[\frac{u^{2}+2}{u \sqrt{4+u^{2}}} \pm 1\right] .
$$

The combined amplification can be written as

$$
A=A_{+}+A_{-}=\frac{u^{2}+2}{u \sqrt{4+u^{2}}} .
$$

For $u \ll 1$, the combined amplification can be approximated as

$$
A \simeq \frac{1}{u}
$$

and for $u \gg 1$

$$
A \simeq 1+\frac{2}{u^{4}} .
$$

The intensity-weighted centroid of the two images is given by

$$
\theta=\frac{A_{+} \theta_{+}+A_{-} \theta_{-}}{A} .
$$

If the two images are unresolved, then the centroid shift with respect to the original position of the source can now be written as

$$
\delta \theta=\theta-\Delta \theta=\frac{u}{u^{2}+2} \theta_{\mathrm{E}} .
$$

The locations of the two images, and of their centroid, as functions of $u$ are shown in the top panel of Figure 4, and the corresponding magnifications are shown in the bottom panel. Note that $\delta \theta$ increases with $u$ when $u<\sqrt{2}$, after which it decreases with $u$. As the figure shows, when $u \gg 1$ (as in the case of Proxima), $A_{-} \simeq 0$ and $A \simeq A_{+}$, so that the position of the major image can be taken as the centroid of the combined image, and its amplification as the amplification of the combined image.

For $u \gg 1$, the centroid shift can now be written as

$$
\delta \theta \simeq \frac{\theta_{\mathrm{E}}}{u}=\frac{\theta_{\mathrm{E}}^{2}}{\Delta \theta} .
$$

Adopting a distance of $1.3 \mathrm{pc}$ and a mass of $0.12 M_{\odot}$ for Proxima, and assuming $D_{S} \gg D_{L}$ in Equation (1), we find that its Einstein ring radius is $\theta_{E} \simeq 28$ mas. Thus a background source lying at an angular distance $\Delta \theta$ from Proxima will have its apparent position shifted by

$$
\delta \theta \approx \frac{(28 \mathrm{mas})^{2}}{\Delta \theta}
$$



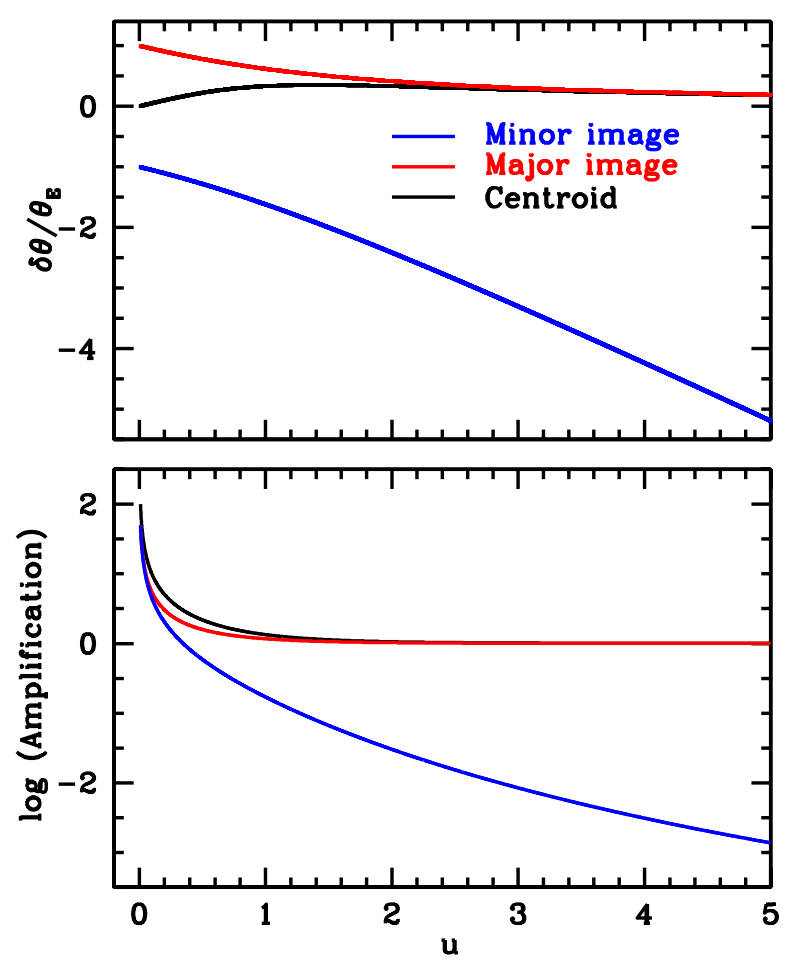

Figure 4. Astrometric shift and the amplifications of the images. The upper panel shows the shifts of the minor image, the major image, and the centroid with respect to the undeflected position of the source, as a function of $u$. The lower panel shows the amplifications of the same three components as a function of $u$. The shift of the minor image monotonically increases with $u$, but its brightness drastically reduces for larger $u$ as seen in the lower panel. As a result, the contribution of the minor image is negligible at high $u$, and the astrometric shift and the amplification are close to that of the major image.

Using Equations (1) and (11), we can express the mass of the lens as

$$
M=\frac{\theta_{\mathrm{E}}^{2} c^{2} d_{\pi}}{4 G} \simeq \frac{\delta \theta c^{2} d_{\pi} \Delta \theta}{4 G} .
$$

The angular separation $\Delta \theta$ and the centroid shift $\delta \theta$ can be determined from the observations taken before and during the event. To measure the mass of the lens using Equation (13), the remaining required parameter is the parallax distance $d_{\pi}$, depending only on the difference between the parallaxes of the lens and the source. The parallax of the lens is already known, and our planned $H S T$ observations will directly constrain the source parallaxes (expected to be very small as noted above).

For the upcoming close encounters of Proxima Centauri, the sources lie on either side, with impact parameters of 0.5 and 1.6 arcsec, which correspond to $u \simeq 18$ and 57, respectively. For such large $u$, the major image will lie within $\sim 1.5$ mas of the undeflected source location. The brightness of the minor image, which will lie within $\sim 1$ mas of Proxima itself, will be $<2 \times 10^{-5}$ that of the primary image, and thus can be ignored.

In Figures 5 and 6 we plot the centroid shifts of Sources 1 and 2 as functions of time, under the assumption that Proxima has a mass of $0.12 M_{\odot}$. The maximum centroid shift for Source 1 is $\sim 0.5$ mas at closest approach, and for Source 2 it is $\sim 1.5$ mas. As seen from Equation (12), the centroid shift scales inversely with the angular separation between Proxima and the source star, so the deflections are already underway as we write! Assuming that the centroid shifts of the two sources can be measured with an accuracy of 200 mas per epoch, Proxima's mass can be measured with an accuracy of $\sim 5 \%$.

\section{ASTROMETRIC DETECTION OF PLANETS AROUND PROXIMA}

If Proxima has planetary companions, it may be possible to detect them-and measure their masses - through the extra astrometric shifts of one or both of the background stars (Safizadeh et al. 1999; Han \& Lee 2002). Using Equation (1), we can write the size of the angular Einstein ring due to a planet around Proxima as

$$
\left(\theta_{\mathrm{E}}\right)_{\text {planet }} \simeq 8\left(\frac{M_{\text {planet }}}{M_{\text {Jup }}}\right)^{1 / 2} \text { mas. }
$$

The maximum possible extra deflection due to a Jupiter-mass planet is $(\sqrt{2} / 4) \theta_{\mathrm{E}} \simeq 2.8$ mas, which would occur when the angular separation between the background source's deflected image and the planet is $\sqrt{2} \theta_{\mathrm{E}} \simeq 11$ mas.

Figure 6 shows an example of how the gravitational deflection of Source 2 might be modified by the presence of a planetary companion of Proxima under favorable circumstances. We have assumed a Jupiter-mass companion in a face-on circular orbit with a separation of $0.8 \mathrm{AU}$, which has a closest approach of 14 mas from the background star. The dashed lines in both panels show the deflections when this hypothetical planet is included in the calculations. The effect of the motion of the planet in its 751 day orbit is included. As the figure illustrates, such a planet would produce a relatively large distortion of the deflection curve by about 0.5 mas, but lasting only about three days.

Let us now calculate the sizes of the regions around Proxima in which planets can be detected through their astrometric signals. If the minimum astrometric deflection that can be measured is $\delta_{\min }$, then the corresponding maximum angular distance between the source and the planet can be written, using Equation (9), as

$$
\phi_{\max }=\frac{\left(\theta_{\mathrm{E}}\right)_{\text {planet }}^{2}}{\delta_{\min }} \simeq \frac{(8 \text { mas })^{2}}{\delta_{\min }}\left(\frac{M_{\text {planet }}}{M_{\text {Jup }}}\right) .
$$

This equation implies, for example, that if $\delta_{\min }=0.03$ mas (detectable with HST spatial scanning as discussed above), the deflection due to a Jupiter-mass planet would be detectable if the planet passes within $2^{\prime \prime}$ of either background source.

Figure 7 illustrates the spatial regions around Proxima in which planets of various masses will be detectable during the upcoming approaches of the two background stars. Here we show the trajectories of the two stars in a Proxima-centered reference frame. Dashed curves refer to Source 1, and solid curves to Source 2 . The sets of blue curves surrounding both trajectories, with separations of $\sim 2^{\prime \prime}$ from the stars, enclose the regions in which a Jupiter-mass planet is detectable with a deflection exceeding 0.03 mas. The red curves surrounding the two trajectories enclose the regions in which the deflection due to a $10 M_{\text {Earth }}$ planet is detectable.

Even though astrometric microlensing provides an exciting possibility of detecting planets around Proxima, the probability of such a detection is small for two reasons. First, the microlensing signal due to a planet lasts only about three days. Second, there is a zone close to Proxima where it becomes difficult to distinguish the signal due to a planet from that due to Proxima itself. Detection of the planetary signal is easier when the planet passes closer to the source star, and is most favorable when the planet passes through a point where its separation from the source is much less than the planet's separation from Proxima. 

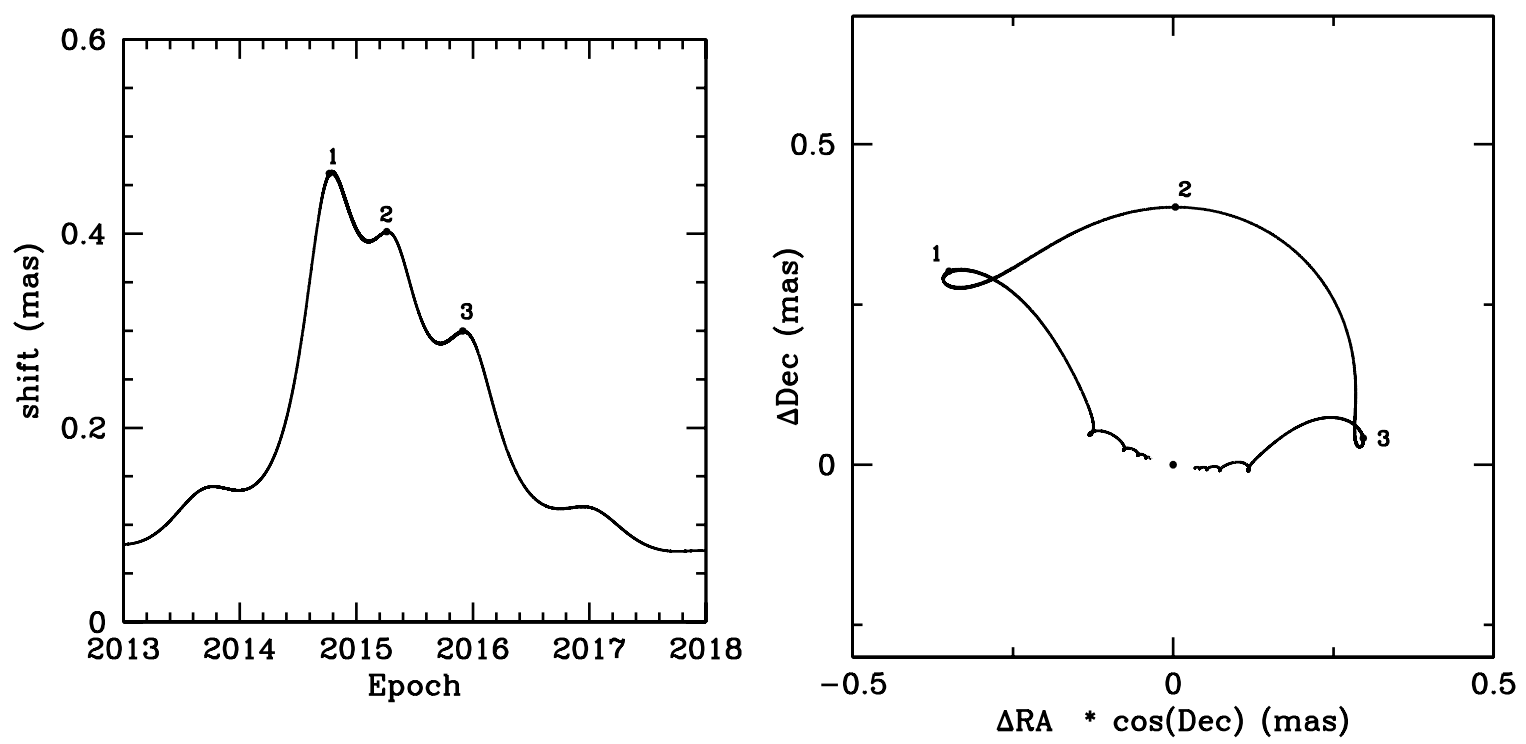

Figure 5. Expected astrometric shifts of Source 1 (the fainter of the two background stars). The left panel shows the total astrometric shift as a function of time, and the right panel shows the two-dimensional motion of the image of the background star. The epochs of three peak deflections are marked with numerals 1,2 , and 3 in the left panel, and the corresponding points are labeled in the right panel.
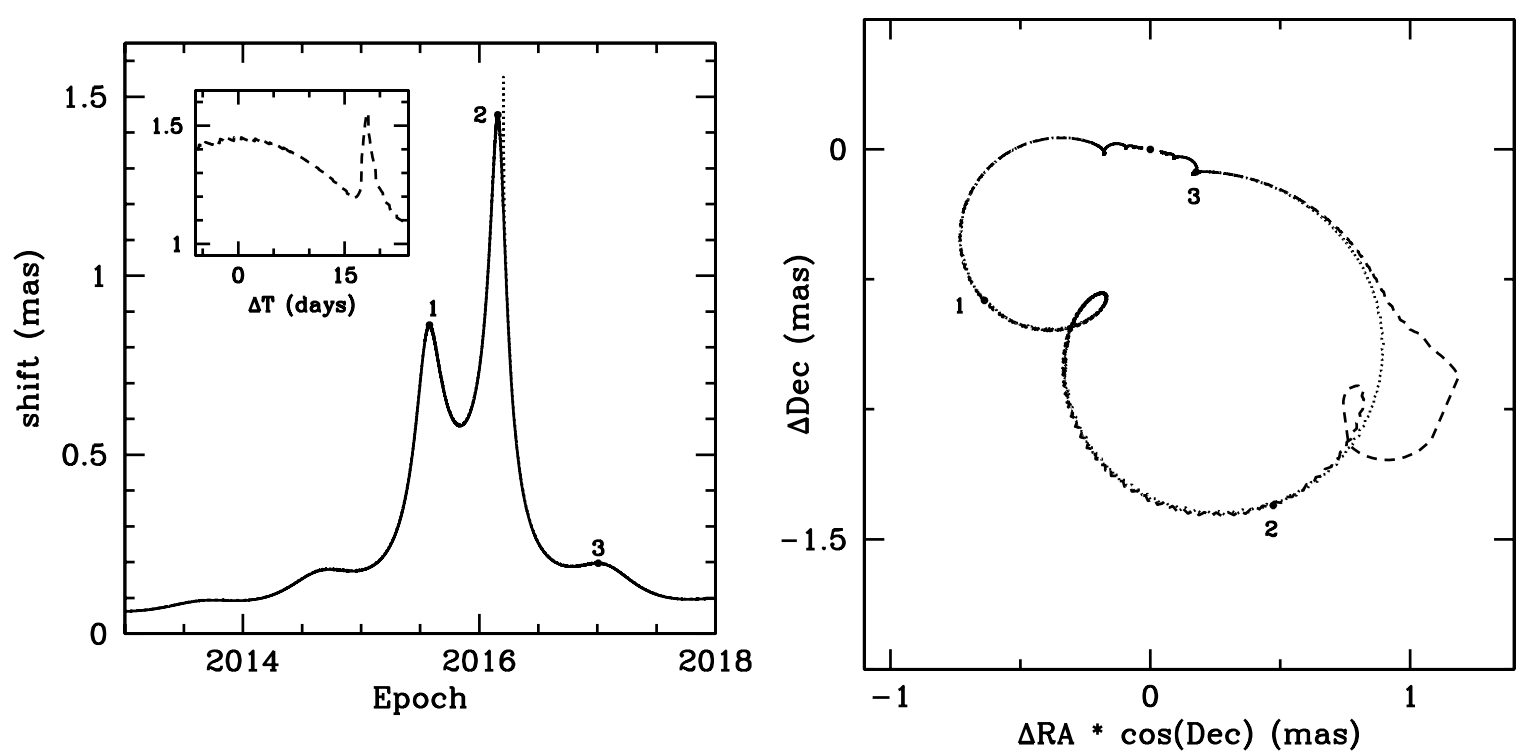

Figure 6. Expected astrometric shifts of Source 2 (the brighter of the two background stars). The solid line in the left panel shows the total astrometric shift as a function of time, and the right panel shows the two-dimensional motion of the image of the background star. The epochs of three peak deflections are marked with numerals 1,2, and 3 in the left panel, and the corresponding points are labeled in the right panel. The dashed lines in both panels show the shifts when we additionally assume Proxima to be accompanied by a Jupiter-mass planet at an orbital separation $0.8 \mathrm{AU}$, which passes within 14 mas of the background star. These calculations take the orbital motion of the planet into account. The inset in the left panel shows a magnified view of the region of the planet's effect on the astrometric shift, which has a duration of about three days.

\section{PHOTOMETRIC MICROLENSING BY PLANETS AROUND PROXIMA}

If Proxima has a planet which passes within a few mas of either source, photometric signatures of microlensing due to the planet may be detectable. As noted earlier, the Einstein ring of a Jupiter-mass planet has a radius of about 8 mas. For a photometric accuracy of $1 \%$, the planetary signature will be detected for an amplification of $A \geqslant 1.01$, which is achieved if the impact parameter $u \leqslant 3.5$ (Equation (4)). This requires the planet to lie within an angular distance $\zeta$ of the source, where

$$
\zeta=3.5\left(\theta_{\mathrm{E}}\right)_{\text {planet }} \simeq 28\left(\frac{M_{\text {planet }}}{M_{\text {Jup }}}\right)^{0.5} \text { mas. }
$$

Thus any Jovian-mass planet that passes within 28 mas of either background star (corresponding to a linear separation of $\leqslant 0.036 \mathrm{AU}$ ) can be detected through precision photometry.

The Einstein-ring crossing time is given by $T_{\mathrm{E}}=\theta_{\mathrm{E}} / A$, where $A$ is the proper motion of the planet relative to the background star. Assuming the proper motion of Proxima for the planet, the crossing time is

$$
\left(T_{E}\right)_{\text {planet }} \simeq 2.7\left(\frac{M_{\text {planet }}}{M_{\text {Jup }}}\right)^{0.5} \text { days. }
$$

The duration of the photometric event can be written as

$$
T_{\mathrm{D}}=2 T_{\mathrm{E}}\left(b^{2}-u^{2}\right)^{0.5}
$$




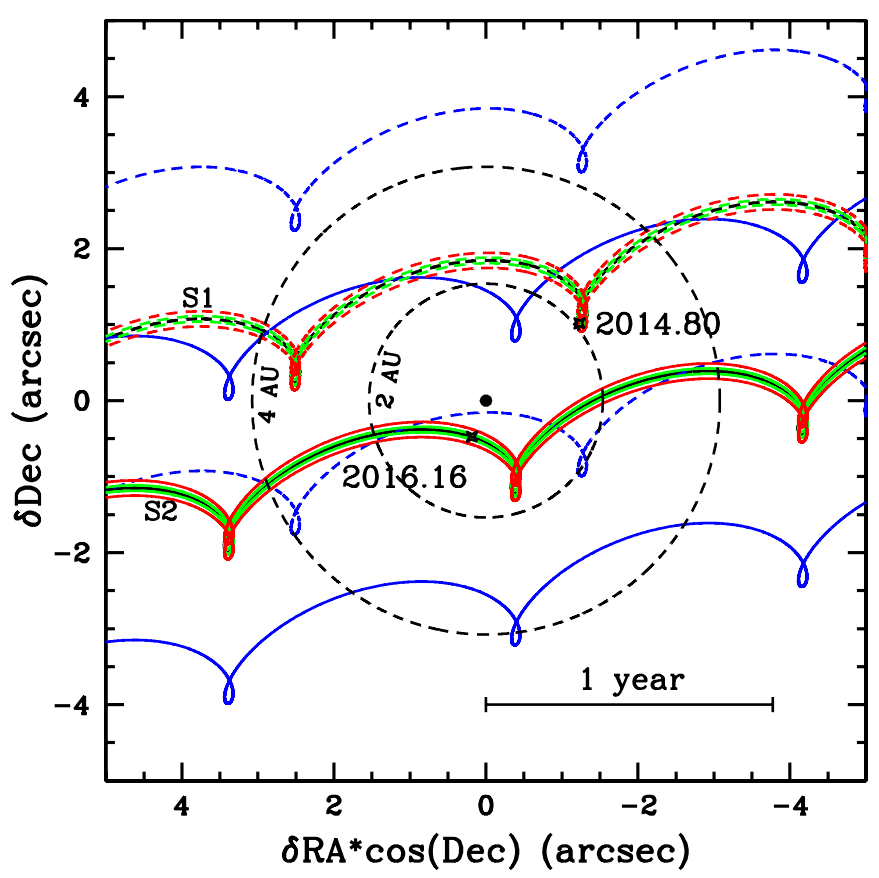

Figure 7. Trajectories of the two background stars in a Proxima-centered reference frame, shown as dashed (Source 1, labeled as "S1") and solid (Source 2, labeled as "S2") black lines. Black dot at the center denotes Proxima, surrounded by two dashed black circles of radii 2 and 4 AU. If Proxima has a $1 M_{\text {Jup }}$ planet lying between the pairs of dashed blue (Source 1) or solid blue (Source 2) lines, the extra astrometric shift caused by the planet will (briefly) exceed 0.03 mas. If Proxima has a $10 M_{\text {Earth }}$ planet lying between the pairs of red lines, the astrometric signal of this planet will likewise exceed 0.03 mas. If Proxima has a $1 M_{\text {Jup }}$ planet within the green lines, its photometric microlensing signal will exceed $0.01 \mathrm{mag}$. The locations and dates of the closest approaches of the two background stars are marked. As they move to the east (left) relative to Proxima, the parallactic loops of the background stars have a $1 \mathrm{yr}$ timescale, as indicated by the scale bar at the bottom. The $x$-axis denotes the absolute value of the shift, i.e., $\delta$ R.A. $\times \cos ($ decl. $)$.

where $b$ is the source-lens separation, in units of $\theta_{\mathrm{E}}$, at which the amplification is detectable. An amplification of $A=1.01$ corresponds to $b=3.5$. Thus $T_{\mathrm{D}}$ can be up to seven times $T_{\mathrm{E}}$, or, for a Jupiter-mass planet, $\sim 19$ days.

In Figure 7 the green lines surrounding each background source trajectory enclose the regions within which a $1 \%$ photometric amplification by a Jovian planet would be detectable. These zones are very small, so the probability of detecting photometric microlensing by a planet is extremely small.

\section{OBSERVATIONAL CONSIDERATIONS}

Successful observations of these events will be challenging, because the background stars are $\geqslant 8.5$ mag fainter than Proxima and will require accurate astrometry and photometry at separations from Proxima ranging from 0.5 to $\sim 2^{\prime \prime}$. Here we discuss the feasibility of the observations from space and from the ground.

\subsection{Space-based Astrometry and Photometry}

For the upcoming events, the minimum values of $\Delta \theta$ will be $1^{\prime \prime} .6$ and 0.5 , corresponding to maximum deflections of 0.5 and 1.5 mas. Such displacements are routinely measured using cameras on board HST; in fact, astrometric accuracies of 0.2 mas have been achieved in single measurements through direct imaging with the Advanced Camera for Surveys and WFC3 (e.g., Anderson \& King 2006; Bellini et al. 2011), and spatialscanning modes have been developed recently with WFC3 that can achieve astrometric accuracies of about 0.03 mas (Riess et al. 2014).

The fact that these observations can be obtained easily with HST even in the presence of Proxima is illustrated in Figure 8. In order to achieve astrometric accuracy of 0.2 mas per observation, we need a signal-to-noise ratio $(\mathrm{S} / \mathrm{N})$ of about 300 for each background star, which can be achieved in an exposure time (WFC3, F555W filter) of $70 \mathrm{~s}$. The left panel shows a $200 \mathrm{~s}$ exposure on Proxima taken with WFC3 in F555W filter, which is thrice the required exposure time. The white circle has a radius of 12.5 pixels $\left(0{ }^{\prime \prime} .5\right)$. The right-hand panel shows the radial profile of this star, greatly magnified to show the signal in the wings of the stellar image. The counts drop below 5000 electrons at a radial distance of 12.5 pixels, at which point the background becomes negligible compared to a star with $\mathrm{S} / \mathrm{N}=$ 300. Since the source star will actually be at a separation of 0.5 even at closest approach, saturation effects are thus unimportant provided the diffraction spikes and charge bleeding are avoided.

These events will conveniently occur during the operational lifetime of Gaia, ESA's astrometry mission, recently successfully launched. Gaia can observe down to $V \simeq 20$, and its astrometric accuracy is expected to be $0.1-0.3$ mas depending on the source magnitude (Gare et al. 2010; Prusti 2012; Liu et al. 2012). Assuming Gaia can observe the fainter background star in the presence of the much brighter Proxima, the mass of Proxima should be measured very well.

\subsection{Ground-based Astrometry and Photometry}

Photometric observations required for this project are possible with several ground-based instruments. One example is the upcoming Spectro-Polarimetric High-contrast Exoplanet REsearch (SPHERE) of ESO Very Large Telescope (VLT), which is specially designed to image faint sources in the presence of sources brighter by as much as 12.5 mag with separations as small as 0'.1 (Beuzit et al. 2008). The Gemini Planet Imager may also be a suitable instrument where a contrast of $>10^{6}$ at 0.4 , and closed-loop performance down to $I=8 \mathrm{mag}$, have been achieved (Macintosh et al. 2012). The "lucky imaging system" at the Danish $1.54 \mathrm{~m}$ telescope at the ESO La Silla Observatory may also be a capable instrument, which uses a Electron-multiplying Charge Coupled Device that delivers fast readout times and negligible readout noise, making it an ideal detector where a large number of fast frames can be used in "shift and add" mode to improve resolution and avoid saturation (Harpsøe et al. 2012).

Astrometric observations from the ground are, however, very challenging. Astrometric accuracies of 0.1 mas have been achieved for stars brighter than $K=16$ with $\mathrm{NaCo}$ (Fritz et al. 2010; Lenzen et al. 2003; Rousset et al. 2003), which is a near-IR imager and spectrograph with an adaptive optics system at ESO VLT. Astrometric measurements with an accuracy of 0.1 mas for sources brighter than $K=16$ have also been achieved with Keck (Clarkson et al. 2012), although Proxima's declination of $-62^{\circ}$ makes it unobservable. However, apart from the fact that both our sources are most likely fainter than $K=16$, they will be accompanied by a very close and much brighter star. In principle, the brighter star can help by serving as a good guide/reference star, but in practice, the "halo" of the bright star is likely to overlap with the position of the target star (Fritz et al. 2010), posing challenges by causing systematics which need to be characterized extremely well. 

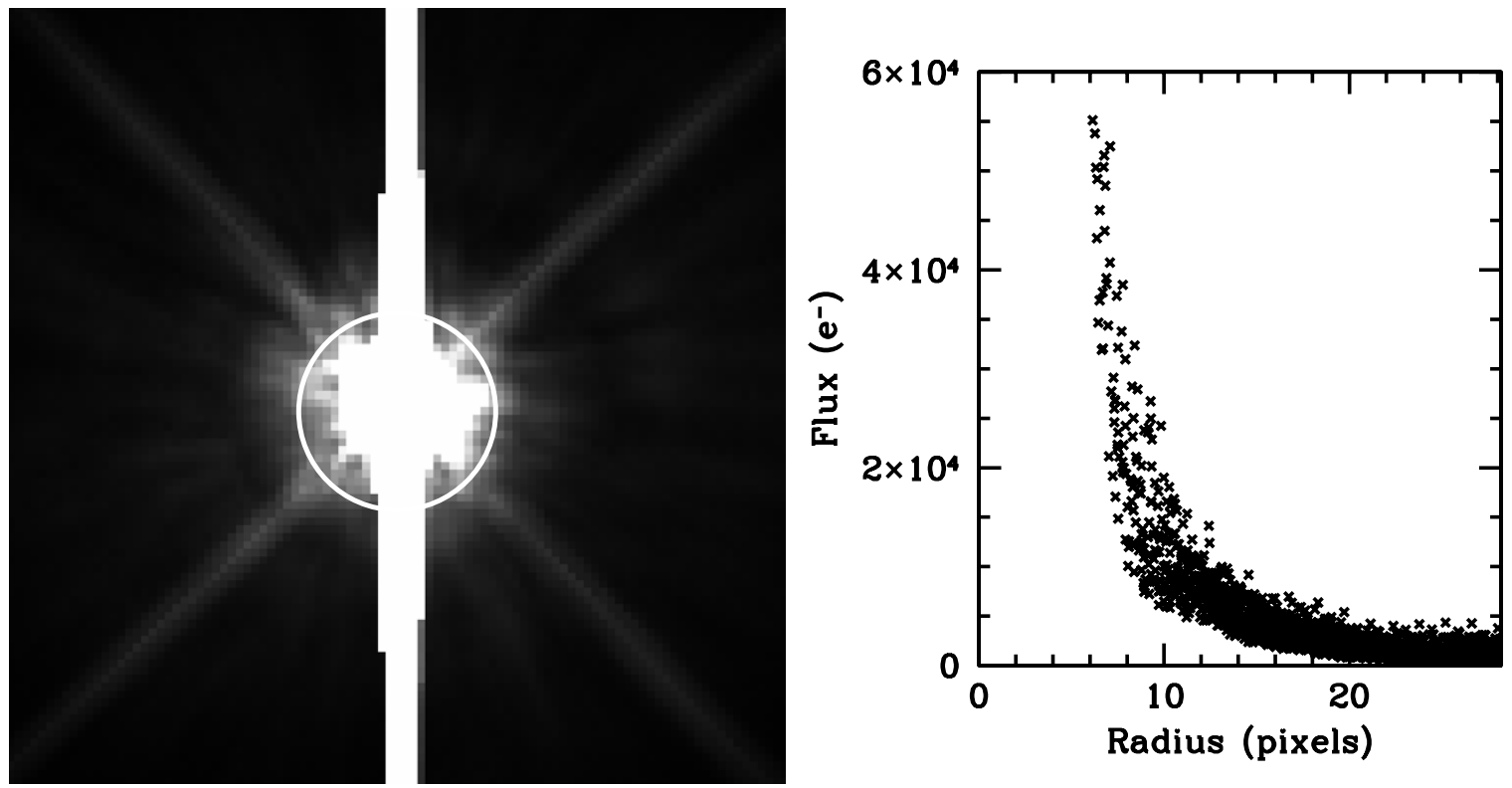

Figure 8. Left: image of Proxima Centauri taken with WFC3/HST on 2012 October 1, in the F555W filter with an exposure time of $200 \mathrm{~s}$. This is about three times overexposed than what is required in order to attain a $\mathrm{S} / \mathrm{N}$ of 300 for even the fainter background star. The white circle corresponds to a radius of 12.5 pixels corresponding to $0.5 \mathrm{arcsec}$, which is the closest approach of the background star. Right: radial profile of Proxima in the $200 \mathrm{~s}$ image, greatly magnified to show the signal in the wings of the stellar image. The counts drop below 5000 electrons at a radial distance of 0.4 , at which point the background becomes small compared to a star with $\mathrm{S} / \mathrm{N}=300$. Since the source star will actually be at a separation of $0{ }^{\prime \prime} 5$ at closest approach, it is important to choose an orientation of the camera such that the sources do not fall either on the direction of charge bleeding or on the diffraction spikes.

\section{SUMMARY}

We have shown that the nearest star, Proxima Centauri, will pass close to two background stars in 2014 and 2016, with impact parameters of about 1".6 and 0.'5. Because Proxima is so near, its angular Einstein ring radius is large ( 28 mas) and will lead to detectable relativistic light deflections of the images of the background stars even at those angular separations. Measurement of the astrometric shifts offers a unique opportunity for the determination of the mass of Proxima with an accuracy of $\sim 5 \%$.

Although the background stars are $\geqslant 8.5$ mag fainter than Proxima, the large contrast is mitigated by the relatively large separations at which the gravitational deflection is still detectable, and well within the capabilities of the HST.

The upcoming events also offer the opportunity to detect and determine the masses of planetary companions, either through additional astrometric shifts, or in rare circumstances through a photometric microlensing event, leading to a brightening of the source star. These events would have durations of a few hours to several days.

We thank Rémi Soummer, Will Clarkson, and Rosanne Di Stefano for useful discussions. Partial support for this research was provided by NASA through grant GO-12985 from the Space Telescope Science Institute, which is operated by the Association of Universities for Research in Astronomy, Inc., under NASA contract NAS5-26555. K.S. acknowledges support from the European Southern Observatory, the Institute for Theory and Computation at the Harvard-Smithsonian Center for Astrophysics, and the Institute for Advanced Study at Princeton for sabbatical visits, during which parts of this work were carried out. M.D. is thankful to the Qatar National Research Fund
(QNRF), member of the Qatar Foundation, for support through grant NPRP 09-476-1-078.

Facility: HST (WFC3)

\section{REFERENCES}

Anderson, J., \& King, I. R. 2006, Instrument Science Report ACS 2006-01 (Baltimore, MD: STSci), 1

Bakos, G., Sahu, K. C., \& Nemeth, P. 2002, ApJS, 141, 187

Bellini, A., Anderson, J., \& Bedin, L. R. 2011, PASP, 123, 622

Benedict, G. F., McArthur, B., Chappell, D. W., et al. 1999, AJ, 118, 1086

Beuzit, J.-L., Feldt, M., Dohlen, K., et al. 2008, Proc. SPIE, 7014, 701418

Campbell, B., Yang, S., Irwin, A. W., \& Walker, G. A. H. 1991, in Proceedings of the Third International Symposium on Bioastronomy, Bioastronomy: The Search for Extraterrestrial Life-The Exploration Broadens, ed. J. Heidmann $\&$ M. J. Klein (Lecture Notes in Physics, Vol. 390; Berlin: Springer), 19

Chabrier, G., \& Baraffe, I. 1997, A\&A, 327, 1039

Chabrier, G., \& Baraffe, I. 2000, ARA\&A, 38, 337

Clarkson, W. I., Ghez, A. M., Morris, M. R., et al. 2012, ApJ, 751, 132

Cutri, R. M., Skrutskie, M. F., van Dyk, S., et al. 2003, The IRSA 2MASS All-Sky Point Source Catalog, NASA/IPAC Infrared Science Archive, http://irsa.ipac.caltech.edu/applications/Gator/

Di Stefano, R. 2008, ApJ, 684, 46

Dominik, M., \& Sahu, K. C. 2000, ApJ, 534, 213

Dumusque, X., Pepe, F, Lovis, C., et al. 2012, Natur, 491, 207

Endl, M., \& Kürster, M. 2008, A\&A, 488, 1149

ESA 1997, The Hipparcos and Tycho Catalogues (ESA SP-1200; Noordwijk: ESA)

Fritz, T., Gillessen, S., Trippe, S., et al. 2010, MNRAS, 401, 1177

Gare, P., Sarri, G., \& Schmidt, R. 2010, ESABu, 137, 51

Gilliland, R. L. 1994, ApJL, 435, L63

Han, C., \& Lee, C. 2002, MNRAS, 329, 163

Harpsøe, K. B. W., Jørgensen, U. G., Andersen, M. I., \& Grundahl, F. 2012, A\&A, 542, A23

Kraus, A. L., Tucker, R. A., Thompson, M. I., Craine, E. R., \& Hillenbrand, L. A. 2011, ApJ, 728, 48

Lasker, B. M., Lattanzi, M. G., McLean, B. J., et al. 2008, AJ, 136, 735

Lenzen, R., Hartung, M., Brandner, W., et al. 2003, Proc. SPIE, 4841, 944

Lépine, S., \& Di Stefano, R. 2012, ApJL, 749, L6 
Lépine, S., \& Shara, M. M. 2005, AJ, 129, 1483

Liu, C., Bailer-Jones, C. A. L., Sordo, R., et al. 2012, MNRAS, 426, 2463

Luyten, W. J. 1979, LHS Catalog (Minneapolis, MN: Univ. Minnesota)

Macintosh, B. A., Anthony, A., Atwood, J., et al. 2012, Proc. SPIE, 8446, $84461 \mathrm{U}$

Paczyński, B. 1996, AcA, 46, 291

Proft, S., Demleitner, M., \& Wambsganss, J. 2011, A\&A, 536, A50

Prusti, T. 2012, AN, 333, 453

Riess, A. G., Casertano, S., Anderson, J., Mackenty, J., \& Filippenko, A. V. 2014, arXiv: 1401.0484
Rousset, G., Lacombe, F., Puget, P., et al. 2003, Proc. SPIE, 4839, 140

Safizadeh, N., Dalal, N., \& Griest, K. 1999, ApJ, 522, 512

Salim, S., \& Gould, A. 2000, ApJ, 539, 241

Schneider, P., Ehlers, J., \& Falco, E. E. 1992, Gravitational Lenses (Berlin: Springer)

Ségransan, D., Kervella, P., Forveille, T., \& Queloz, D. 2003, A\&A, 397, L5

Torres, C. A.O., Quast, G. R., da Silva, L., et al. 2006, A\&A, 460, 695

van Leeuwen, F. 2007, A\&A, 474, 653

Wertheimer, J. G., \& Laughlin, G. 2006, AJ, 132, 1995

Wright, J. T. 2005, PASP, 117, 657 